\title{
Epileptic Seizure Prediction using Power Spectrum and Amplitude Analysis of Beta Band of EEG Signals
}

\author{
Raj Sadaye \\ Dwarkadas J. Sanghvi \\ College of Engineering \\ Mumbai,India.
}

\author{
Sagar Parekh \\ Dwarkadas J. Sanghvi \\ College of Engineering \\ Mumbai,India.
}

\author{
Jeremy Samuel \\ Dwarkadas J. Sanghvi \\ College of Engineering \\ Mumbai,India.
}

\author{
Khushali Deulkar \\ Dwarkadas J. Sanghvi \\ College of Engineering \\ Mumbai,India.
}

\begin{abstract}
Epilepsy is a spectrum condition which varies from person-toperson. It is characterized by unpredictable seizures which cause severe health related problems for the person concerned. Recent research works have focused on predicting epileptic seizures by analysis of frequency band of Electroencephalogram (EEG) signals. This paper proposes a new seizure prediction method by using frequency and amplitude analysis of beta band in EEG signals. In this method, the power spectrum of the beta band of EEG signal is calculated. The power value generated is then combined with the amplitude of the EEG signal and classified using SVM (Support Vector Machine) to achieve efficient and accurate results. Data of 39 patients in the pre-ictal and inter-ictal period was tested using this method. The method used predicts seizures with an accuracy of $70 \%$.
\end{abstract}

\section{Keywords}

EEG; Epileptic Seizure; Beta band; Power Spectrum; SVM

\section{INTRODUCTION}

Epilepsy is the fourth most common neurological disease next to only, migraine, stroke and Alzheimer's disease and about $1 \%$ of the people worldwide have epilepsy. A Seizure is a sudden, intensive, unexplained and unknown neurological activity in the brain [4] .In epilepsy; there are spontaneous seizures which may last for few seconds up to 2 minutes. The different frequency bands act as the source for the feature extraction which is used in different methods. The Beta band of the waves can be extracted by a first sampling of the EEG waves using FFT and Band Pass filter. After obtaining the beta band the feature extraction is done. In feature extraction, the power and amplitude of beta bands are calculated. After calculation of the power, Support Vector Machine algorithm is used for classifying and deciding whether the signal in question is prone to an epileptic seizure in future.

The same approach can be used for the detection and prediction of other mental and psychiatric disorders [1]

But for some epileptic patient seizure cannot be controlled by the medications alone. For some of these patients, surgery is the only option. For surgery, it is required to localize the particular region of the brain responsible for epileptic seizure [1]

The effect of medication can be increased if these are administered at the earliest possible time of the seizure occurrence. Epileptic seizures can be controlled effectively by use of appropriate medications but about $30 \%$ of the people suffering from epilepsy lack seizure control at the proper time So, this seizure prediction can help the epileptic patient a lot.[3]
Seizure forecasting systems hold promise for improving the quality of life for patients with epilepsy. Due to the prediction of an epileptic seizure at such an early stage the effects of medication can be increased and more patients can be treated accordingly.

In order to predict the epileptic seizure the brain activity of patients is classified into two categories, pre-ictal and interictal patterns. The ictal and post-ictal states are repudiated from the classification since these states are used to detect the undergoing seizures and thus provide very little information about the seizures that could occur in near future.

Many studies have been carried out for prediction of seizures. One method by Aarti Sharma [5] suggests a method of calculating power in the beta band of the EEG signal. By dividing the brain into zones power is calculated in each zone. The zone in which there is the highest change in power is held responsible for the seizure. Another method by M. Z. Parvez and M. Paul.[4] proposes using two features viz. frequency and amplitude through DWT decomposition technique and classification using LS-SVM. This method uses the gamma band of brain waves for prediction of epilepsy.Another method by M. Bandarabadi et al. [7] considers the spectral power of 5 EEG bands and 6 channels. The combination of these 30 features was used to create a 435-dimensional feature space. Classification using the Support Vector Machines algorithm was performed and the output is compared with results obtained by the minimum redundancy maximum relevance (mRMR) method The data is then used to predict if a seizure can occur.

\section{DATASET}

Three datasets have been used for training and prediction. The first dataset that is used for training includes data of 5 patients where 3 are known to have epilepsy and 2 of them don't [10]. The second dataset has data of 10 patients containing around 100 signals in total [9]. The third dataset is the EEG dataset of epileptic patients available on Physio.Net website [8]. EEG signals available on this website are recorded using 23 electrode placement, positioned according to International 1020 system [8]. Recordings, accumulated into 24 cases, were collected from 23 subjects of which 5 were males (ages 3-22), 17 were females (ages 1.5-19) and 1 unknown. Most files consist of 23 EEG channels ( 24 or 26 in a few cases). Overall, this 23 patient dataset has 916 hours of continuously recorded EEG and 198 seizures. Sampling frequency for all EEG signals was $256 \mathrm{~Hz}$ with 16-bit resolution. The line frequency of $60 \mathrm{~Hz}$ was removed from the database while recording hence line noise removal is not required on this dataset. Available recordings have been analyzed on an hourly basis in this work and hence duration of EEG signal considered is 3600 seconds. Since sampling rate is $256 \mathrm{~Hz}$, total length of 
signal analyzed in this work at a time comprises of 921600 samples or discrete values.

\section{METHODOLOGY}

\subsection{Separation of Beta frequency bands}

Beta band having frequency range of [14-30 Hz] can be extracted by first sampling and then using the band pass filter on the EEG signal

Fig. 1. EEG waves before filtering

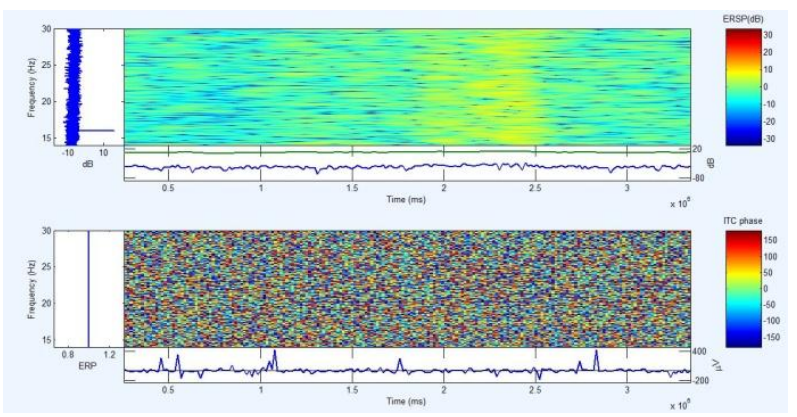

Fig. 2. EEG waves after sampling and filtering

\subsection{Feature selection based on power}

Once the beta frequency band is obtained the power is calculated by using the formula given in equation (1).

$P_{X}=\frac{1}{N} \sum_{N=0}^{N-1}[x(n)]^{2}$

Where $\mathrm{x}(\mathrm{n})$ is the EEG signal, Px is the calculated power and $\mathrm{N}$ is the length of the EEG signals.

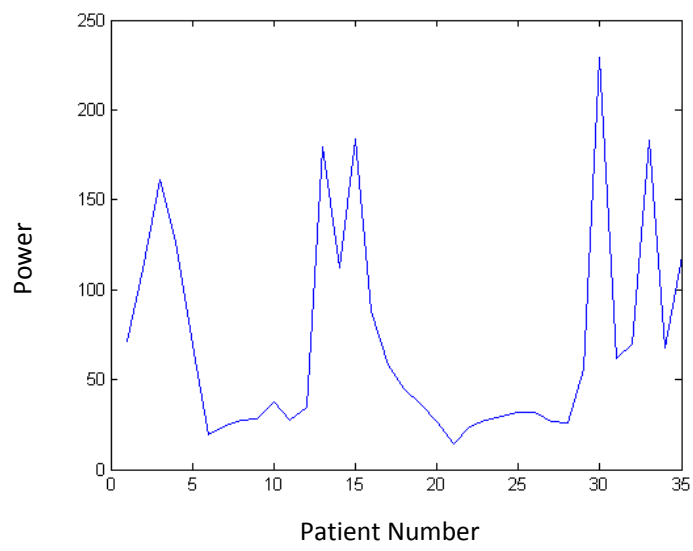

Fig. 3. Beta Band Power

\subsection{Feature selection based on Amplitude Distribution}

After this the maximum amplitude of the EEG signals is calculated. In this approach histograms are plotted for the amplitude distribution of the waves. Histograms are plotted of inter-ictal and pre-ictal samples separately. Using these maximum differences are calculated and then compared with other samples. Also the common area between the pre-ictal and inter-ictal samples of the same patient is identified.

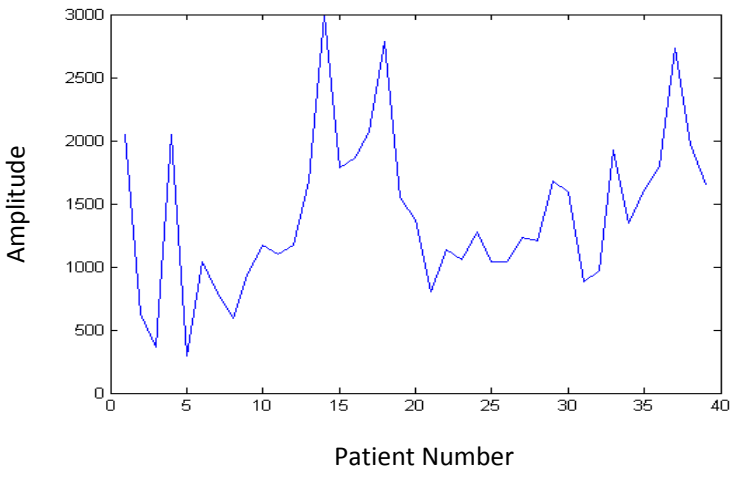

Fig. 4. Maximum Amplitude

\section{ALGORITHM}

Research and observations related to neural activity synchronization, have shown that inter-ictal phases correspond to moderate synchronization within the brain at bands with larger frequency. Thus by observing changes between the pre-ictal and inter-ictal features the onset of a seizure can be predicted. In pre-ictal stage, the beta range synchronization between the epileptic focus and other brain areas decreases.[8]Seizure generation starts in the pre-ictal period and can be observed by a rise in power in beta band.

Fig. 5. illustrates the power of 20 samples in their pre-ictal and inter-ictal period. The sudden rise in the graph denotes the possibility of an epileptic seizure

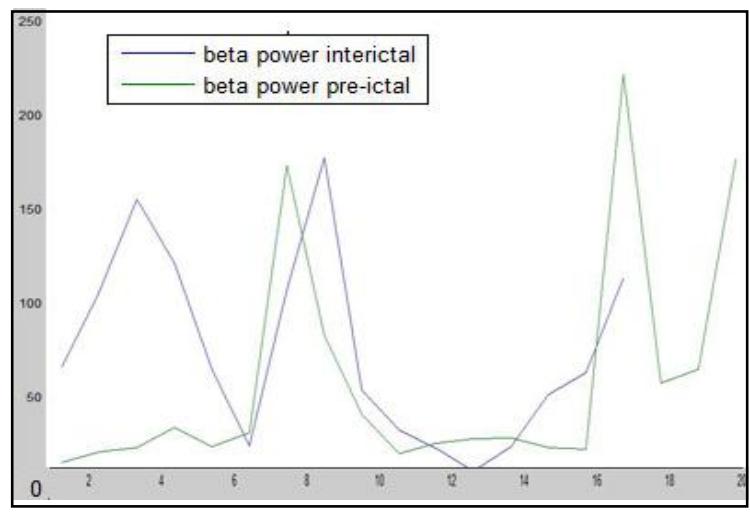

Fig. 5. Power calculations in pre ictal and inter-ictal period

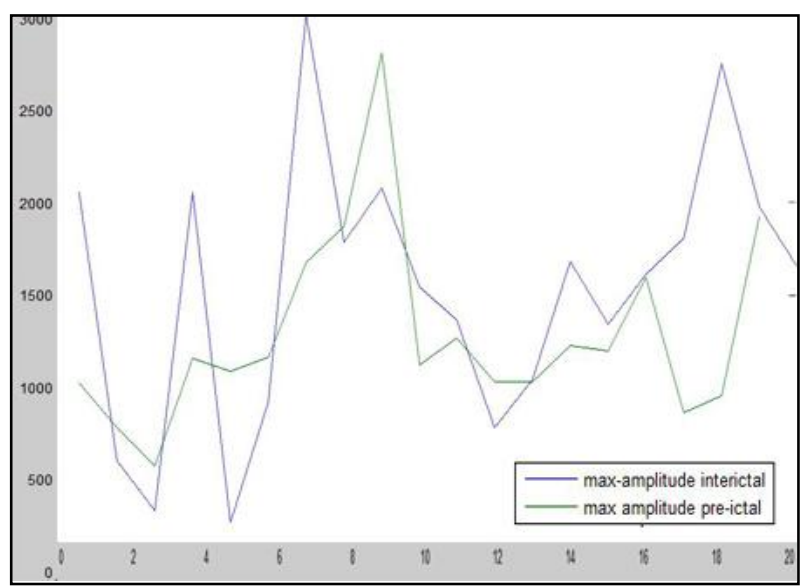

Fig. 6. Amplitude calculations in pre-ictal and inter-ictal period 
The main steps for the implementation of the algorithm are stated below:

The Algorithm Proceeds as follows:

1. The EEG signal is sampled at a frequency of 256 $\mathrm{Hz}$.

2. To convert to a frequency domain signal Fast Fourier Transform is applied to the sampled signal.

3. By applying a band pass filter, extract the beta band of the EEG signal.

4. Calculate the power of beta band of EEG signal.

5. Calculate Maximum amplitude of signal in pre-ictal and inter-ictal period.

6. Feed the values to an SVM classifier.

7. High power values and unusually high amplitude changes are precursors of seizures.

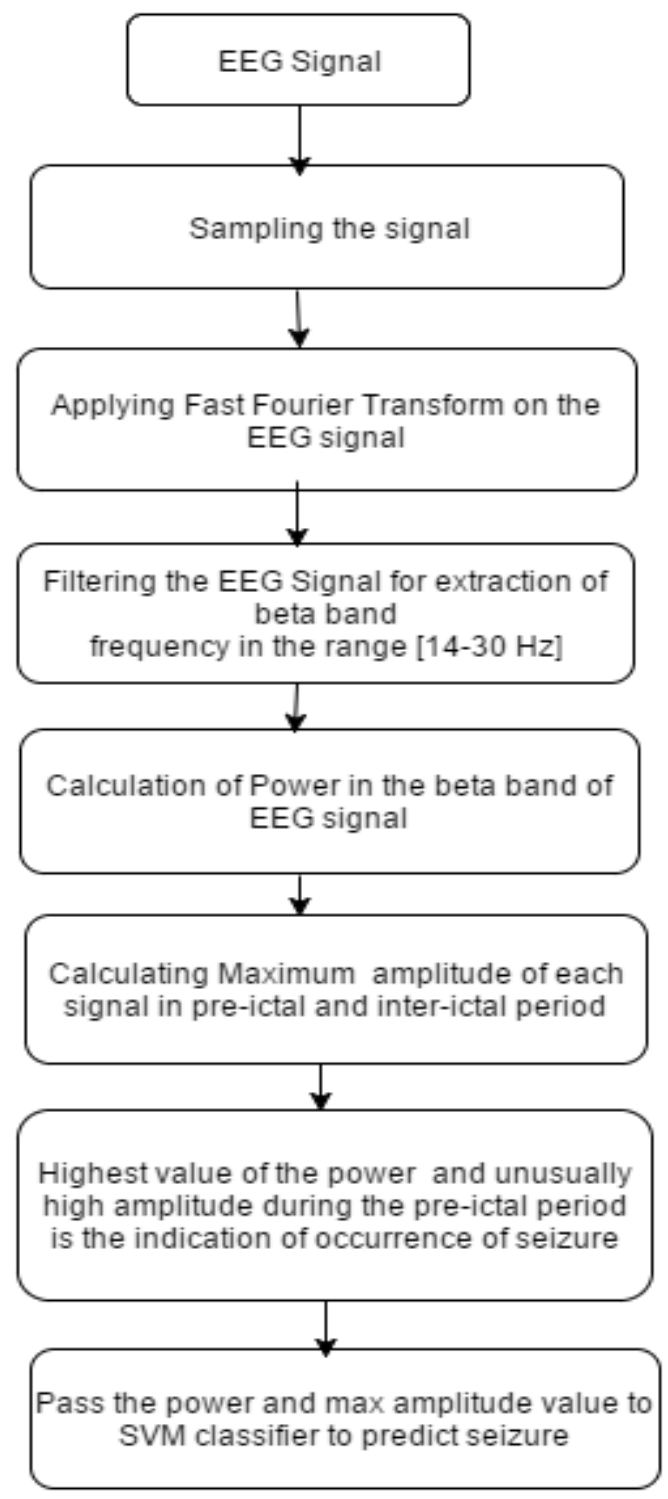

\section{RESULTS AND DISCUSSIONS}

The power and amplitude of the beta band in the pre-ictal and the inter-ictal period have been calculated for 10 patients in MATLAB. The beta band frequencies have been extracted from the EEG signals using bandpass filter.

The seizures are originated during the small window in the pre-ictal period. From Fig.7 and Fig.8. the chances of occurrence of seizure can be predicted effectively. There are possibilities of errors when predicted manually so automatic computerized analysis is required. For analysis the value of power and amplitude is calculated for 10 patients. The results are shown in Table I .From the results it is clear that power in the pre-ictal stage is high enough to predict epileptic seizure.

For patient 1 the value of power in the pre-ictal stage is 19.07433276 and in inter-ictal stage is 71.06046953 . Similarly for patients $2,3,4,7$ and 8 , the value of power in inter-ictal stage rises abruptly from the power value in the pre-ictal stage. Hence the seizure can be predicted in advance and timely medication can be given to the patient.

\begin{tabular}{|c|c|c|c|}
\hline & State & $\begin{array}{l}\text { Maximum } \\
\text { Power(in } \\
\text { decibels) }\end{array}$ & $\begin{array}{l}\text { Maximum } \\
\text { Amplitude( in } \\
\text { microvolts) }\end{array}$ \\
\hline \multirow[t]{2}{*}{ Patient 1} & Pre-ictal & 19.07433276 & 1037.948718 \\
\hline & Inter-ictal & 71.06046953 & 2047 \\
\hline \multirow[t]{2}{*}{ Patient 2} & Pre-ictal & 24.76545666 & 798.046398 \\
\hline & Inter-ictal & 111.2716687 & 623 \\
\hline \multirow[t]{2}{*}{ Patient 3} & Pre-ictal & 27.12782245 & 595.2625153 \\
\hline & Inter-ictal & 161.2469598 & 360 \\
\hline \multirow[t]{2}{*}{ Patient 4} & Pre-ictal & 37.84392263 & 1167.277167 \\
\hline & Inter-ictal & 126.7993519 & 2047 \\
\hline \multirow[t]{2}{*}{ Patient 5} & Pre-ictal & 27.60846706 & 1098.901099 \\
\hline & Inter-ictal & 69.37256363 & 294 \\
\hline \multirow[t]{2}{*}{ Patient 6} & Pre-ictal & 34.87265533 & 1174.310134 \\
\hline & Inter-ictal & 27.81039464 & 936.3614164 \\
\hline \multirow[t]{2}{*}{ Patient 7} & Pre-ictal & 179.752441 & 1677.557998 \\
\hline & Inter-ictal & 112.5202202 & 2992.722833 \\
\hline Patient 8 & Pre-ictal & 87.86855258 & 1867.057387 \\
\hline
\end{tabular}




\begin{tabular}{|c|c|c|c|}
\hline & Inter-ictal & 183.6603113 & 1783.833944 \\
\hline Patient 9 & Pre-ictal & 44.9679883 & 2786.813187 \\
\hline & Inter-ictal & 58.16560539 & 2071.013431 \\
\hline Patient 10 & Pre-ictal & 23.65861895 & 1134.847375 \\
\hline & Inter-ictal & 36.43828241 & 1543.931624 \\
\hline
\end{tabular}

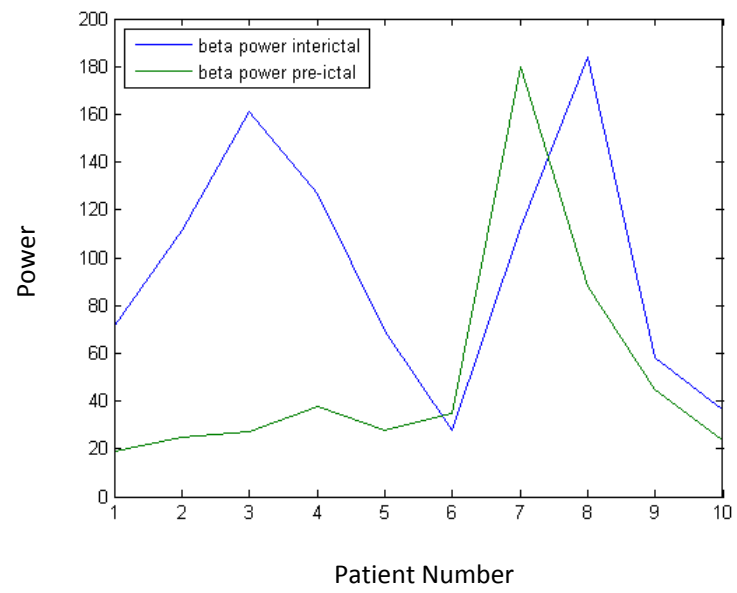

Fig. 7. Power calculations for 10 patients

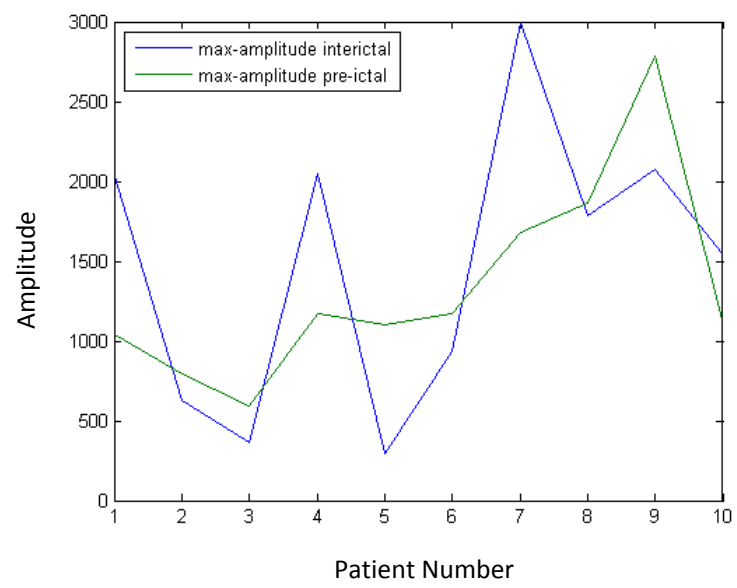

Fig. 8. Amplitude calculations for 10 patients

The algorithm was run for 39 patients with their pre-ictal and inter-ictal data. A Linear Support Vector Machine Algorithm was used to classify the patients based on their power and maximum amplitude values. The prediction output is illustrated in figure 9 .The values on the right of the line are the patients for whom the algorithm predicts a seizure in future. The values on the left of the line are the ones where the algorithm predicts that seizure will not occur.

The algorithms predicts seizure successfully with an accuracy of $70 \%$.The sensitivity of the algorithm is $66.66 \%$. The specificity of the algorithm is $25 \%$. The accuracy of the algorithms will increase with the number of patients that can be incorporate in the classification phase
The SVM kernel is trained in such a way that a prediction is made considering both power and amplitude. As we can see in Figure 10, there are cases where the amplitude rises abruptly, but it is not accompanied by a high raise in beta band power. Hence the SVM algorithm predicts it to be a non-seizure case. These abnormalities in amplitude can be caused when the probes of the EEG sensors move while reading brain waves. Combining features of more than one frequency bands will lead to better classification and prediction.

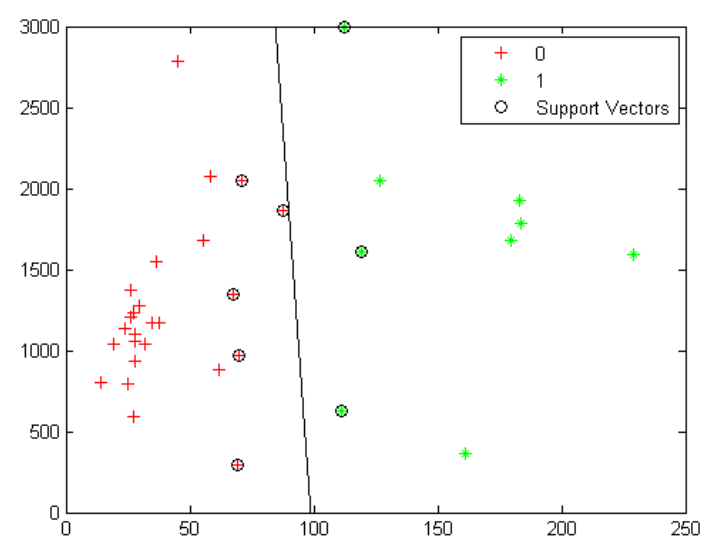

Fig.10: SVM Output of 39 patients

\section{CONCLUSION}

This paper proposes a novel approach to predict epileptic seizure using beta band power and amplitude analysis of the EEG signal. By analysing the signal in the pre-ictal period the algorithm can predict the occurrence of a seizure by observing sudden changes in values between the pre-ictal and inter-ictal period. Analysis using the above stated algorithm on the EEG data has shown successful and promising results. Future research will involve using different kernels of SVM as well as removing noisy classifications while detecting pre-ictal signals for a better and accurate prediction of result. Also the algorithm can be improved by incorporating features from alpha, gamma and theta band of EEG signal for predicting epileptic seizures. A larger dataset and use of neural networks could be investigated to improvise the prediction of results. Another area of future research would involve analysing various features such as frequency, power, spectral power, amplitude etc. and their effects on efficient prediction of epileptic seizures. The future scope of this research is to develop a system which can predict seizures accurately and in advance, thus being able to provide medication and appropriate help in time to reduce the patient's suffering during the seizure.

\section{REFERENCES}

[1] J. Dauwels, F. Vialatte and A. Cichocki, "Diagnosis of Alzheimer's Disease from EEG Signals: Where Are We Standing?", Current Alzheimer Research, vol. 7, no. 6, pp. 487-505, 2010.

[2] J. Dauwels, E. Eskandar and S. Cash, "Localization of seizure onset area from intracranial non-seizure EEG by exploiting locally enhanced synchrony", Proc. IEEE Int. Conf. on Signal Processing EMBS 2009,Minnesota, USA, 2180-2183

[3] S. Ramgopal, S. T. Souza, M. Jackson and T. Loddenkemper, "Seizure detection, seizure prediction 
and closed loop warning system in epilepsy”, Epilepsy and Behavior ,vol. 37, pp. 291-307, 2014.

[4] M. Z. Parvez and M. Paul, "EEG signal classification using frequency band analysis towards epileptic seizure prediction," Computer and Information Technology (ICCIT), 2013 16th International Conference on, Khulna, 2014, pp. 126-130. doi: 10.1109/ICCITechn.2014.6997315

[5] A. Sharma, "Epileptic seizure prediction using power analysis in beta band of EEG signals," 2015 International Conference on Soft Computing Techniques and Implementations (ICSCTI), Faridabad, 2015, pp. 117121. doi: 10.1109/ICSCTI.2015.7489552

[6] M. Bandarabadi, C. A. Teixeira, B. Direito and A. Dourado, "Epileptic seizure prediction based on a bivariate spectral power methodology," 2012 Annual International Conference of the IEEE Engineering in Medicine and Biology Society, San Diego, CA, 2012, pp. 5943-5946. doi: 10.1109/EMBC.2012.6347347
[7] Yadollahpour A, Jalilifar M. Seizure Prediction Methods: A Review of the Current Predicting Techniques. Biomed Pharmacol J 2014;7(1)

[8] Andrzejak RG, Lehnertz K, Rieke C, Mormann F, David P, Elger CE (2001) Indications of nonlinear deterministic and finite dimensional structures in time series of brain electrical activity: Dependence on recording region and brain state, Phys. Rev. E, 64, 061907,

[9] Andrzejak RG, Schindler K, Rummel C (2012). Nonrandomness, nonlinear dependence, and nonstationarity of electroencephalographic recordings from epilepsy patients. Phys. Rev. E, 86, 046206

[10] Physionet_chbmit/physionet.org/physiobank.

[11] V. Jurack, D. Tsuzuki and I. Dau, "10/20, 10/10 and 10/5 system revisited: Their validity as relative head surface based positioning system", Neuroimage, vol. 34, pp. 1600-1610, 2007. 